(C) 2004 International Press

Adv. Theor. Math. Phys. 8 (2004) 177-188

\title{
Matroids and p-Branes
}

\author{
J. A. Nieto \\ Departamento de Investigación en Física de la Universidad de Sonora, \\ 83190, Hermosillo Sonora, México. \\ and \\ Facultad de Ciencias Físico-Matemáticas de la Universidad Autónoma de \\ Sinaloa, 80010, Culiacán Sinaloa, México. ${ }^{1}$ \\ nieto@uas.uasnet.mx
}

\begin{abstract}
A link between matroid theory and $p$-branes is discussed. The Schild type action for $p$-branes and matroid bundle notion provide the two central structures for such a link. We use such a connection to bring the duality concept in matroid theory to $p$-branes physics. Our analysis may be of particular interest in M-theory and in matroid bundle theory.
\end{abstract}

e-print archive: http://lanl.arXiv.org/abs/hep-th/0310071

${ }^{1}$ Permanent address 
The matroid bundle mathematical structure [1]-[3] emerged as a natural extension of oriented matroid theory [4]. Part of the mathematical motivation for such a structure arose when Gelfand and MacPherson [5] discovered a connection between matroid bundle and Pontrjagin classes. Physically, the matroid bundle concept had led to the proposal of a new gravitational theory called gravitoid theory [6]. Moreover, it had been shown [7]-[9] that supergravity $D=11$, Chern-Simons theory and string theory are closely related to matroid bundle.

Here, we are interested in discussing the possibility of linking matroids and $p$-branes via Schild type action [10] (see Ref. [11] and also Refs. [12]-[15]) for $p$-branes and matroid bundle notion. Our analysis may be of particular interest in M-theory [16]-[18] and in matroid bundle theory itself.

Consider a $p$-brane moving in a $d+1$-dimensional Minkowski space-time. We describe the evolution of such a system by the $d+1$-scalar field coordinates $x^{\mu}\left(\xi^{a}\right)$, where $\mu=0,1, \ldots, d$, which are functions of the arbitrary parameters $\xi^{a}$, with $a=0,1, \ldots, p$.

The Dirac-Nambu-Goto type action for $p$-branes is

$$
S_{p}^{(1)}=-T_{p} \int d^{p+1} \xi \sqrt{-h}
$$

where $h \equiv \operatorname{det}\left(h_{a b}\right)$, with

$$
h_{a b}=\partial_{a} x^{\mu} \partial_{b} x^{\nu} \eta_{\mu \nu}
$$

and $T_{p}$ is a fundamental constant measuring the inertia of the $p$-brane. Here,

$$
\eta_{\mu \nu}=\operatorname{diag}(-1,1, \ldots, 1)
$$

is the Minkowski metric.

Let us write $h$ in the form

$$
h=\frac{1}{(p+1) !} \sigma^{\mu_{1} \ldots \mu_{p+1}} \sigma_{\mu_{1} \ldots \mu_{p+1}},
$$

where

$$
\sigma^{\mu_{1} \ldots \mu_{p+1}}=\varepsilon^{a_{1} \ldots a_{p+1}} v_{a_{1}}^{\mu_{1}}(\xi) \ldots v_{a_{p+1}}^{\mu_{p+1}}(\xi) .
$$

Here, $\varepsilon^{a_{1} \ldots a_{p+1}}$ is the totally antisymmetric tensor and 


$$
v_{a}^{\mu}(\xi)=\partial_{a} x^{\mu}(\xi)
$$

It turns out that the action (1) is equivalent to

$$
S_{p}^{(2)}=\int d^{p+1} \xi\left(\sigma^{\mu_{1} \ldots \mu_{p+1}} p_{\mu_{1} \ldots \mu_{p+1}}-\frac{\gamma}{2}\left(p^{\mu_{1} \ldots \mu_{p+1}} p_{\mu_{1} \ldots \mu_{p+1}}+T_{p}^{2}\right)\right),
$$

where $\gamma$ is a lagrange multiplier and the quantity $p_{\mu_{1} \ldots \mu_{p+1}}$ can be understood as the linear momentum associated to $\sigma^{\mu_{1} \ldots \mu_{p+1}}$. Varying (6) with respect to $p_{\mu_{1} \ldots \mu_{p+1}}$ it allows to eliminate $p_{\mu_{1} \ldots \mu_{p+1}}$. We get

$$
S_{p}^{(3)}=\frac{1}{2} \int d^{p+1} \xi\left(\gamma^{-1} \sigma^{\mu_{1} \ldots \mu_{p+1}} \sigma_{\mu_{1} \ldots \mu_{p+1}}-\gamma T_{p}^{2}\right) .
$$

By eliminating $\gamma$ from (7) one recovers the action (1). The importance of (6) or (7) is that it now makes sense to set $T_{p}=0$. In this case (7) is reduced to the Schild type null $p$-brane action [10]-[11].

Here, we are interested in relating (7) to matroid bundle theory. For this purpose it is convenient to recall the definition of an oriented matroid.

An oriented matroid $\mathcal{M}$ is a pair $(S, \chi)$, where $S$ is a non-empty finite set and $\chi$ (called chirotope) is a mapping $S^{r} \rightarrow\{-1,0,1\}$, with $r$ the rank on $S$, satisfying the following properties.

$(\chi i) \chi$ is not identically zero,

$(\chi i i) \chi$ is alternating,

( $\chi$ iii) for all $x_{1}, x_{2}, \ldots, x_{r}, y_{1}, y_{2}, \ldots, y_{r} \in S$ such that

$$
\chi\left(x_{1}, x_{2}, \ldots, x_{r}\right) \chi\left(y_{1}, y_{2}, \ldots, y_{r}\right) \neq 0,
$$

there exists an $i \in\{1,2, \ldots, r\}$ such that

$$
\chi\left(y_{i}, x_{2}, \ldots, x_{r}\right) \chi\left(y_{1}, y_{2}, \ldots, y_{i-1}, x_{1}, y_{i+1}, \ldots, y_{r}\right)=\chi\left(x_{1}, x_{2}, \ldots, x_{r}\right) \chi\left(y_{1}, y_{2}, \ldots, y_{r}\right)
$$

For a vector configuration the chirotope $\chi$ can be identified as

$$
\chi\left(\mu_{1}, \ldots, \mu_{r}\right) \equiv \operatorname{sign} \operatorname{det}\left(b^{\mu_{1}}, \ldots, b^{\mu_{r}}\right) \in\{-1,0,1\},
$$


for all $b^{\mu_{1}}, \ldots, b^{\mu_{r}} \in R^{r}$ and for all $\mu_{1}, \ldots, \mu_{r} \in S$. In this case (10) becomes connected with the Grassmann-Plucker relation (see Ref.. [4], section 3.5).

It can be proved that the definition of the underlying matroid $M$ of $\mathcal{M}$ follows from the chirotope definition for oriented matroids. In fact, from the chirotope definition it follows that if $\mathcal{B}$ is the set of $r$-subsets of $S$ such that

$$
\chi\left(x_{1}, x_{2}, \ldots, x_{r}\right) \neq 0,
$$

for some ordering of $\left(x_{1}, x_{2}, \ldots, x_{r}\right)$ of $\mathcal{B}$, then $\mathcal{B}$ is the set of bases of the matroid $M$. Formally, the definition of $M$ in terms of the bases is as follows (see Ref. [19]):

A matroid $M$ is a pair $(S, \mathcal{B})$, where $S$ is a non-empty finite set and $\mathcal{B}$ is a non-empty collection of subsets of $S$ (called bases) satisfying the following properties:

$(\mathcal{B} i)$ no base properly contains another base;

$(\mathcal{B} i i)$ if $B_{1}$ and $B_{2}$ are bases and if $b$ is any element of $B_{1}$, then there is an element $g$ of $B_{2}$ with the property that $\left(B_{1}-\{b\}\right) \cup\{g\}$ is also a base.

Let us write $(10)$ in the form $\chi\left(\mu_{1}, \ldots, \mu_{r}\right) \equiv \operatorname{sign} \Sigma^{\mu_{1} \ldots \mu_{r}}$, where

$$
\Sigma^{\mu_{1} \ldots \mu_{r}} \equiv \varepsilon^{a_{1} \ldots a_{r}} b_{a_{1}}^{\mu_{1}} \ldots b_{a_{r}}^{\mu_{r}} .
$$

Here, the indices $a_{1}, \ldots, a_{r}$ run from 1 to $r$. Comparing (4) and (12) we observe the great similarity between the two formulae. The main difference comes from the fact that while $v_{a}^{\mu}(\xi)$ is a local object, $b_{a}^{\mu}$ is not. Therefore, our task is to understand the transition from $b_{a}^{\mu}$ to $v_{a}^{\mu}(\xi)$.

Let $\wedge_{r} R^{n}$ denote the $\left(\begin{array}{l}n \\ r\end{array}\right)$-dimensional real vector space of alternating $r$-forms on $R^{n}$. An element $\boldsymbol{\Sigma}$ in $\wedge_{r} R^{n}$ is said to be decomposable if

$$
\Sigma=\mathbf{b}_{1} \wedge \mathbf{b}_{2} \wedge \ldots \wedge \mathbf{b}_{r}
$$

for some $\mathbf{b}_{1}, \mathbf{b}_{2}, \ldots, . \mathbf{b}_{r} \in R^{n}$. It is not difficult to see that (13) can be written as

$$
\boldsymbol{\Sigma}=\frac{1}{r !} \Sigma^{\mu_{1} \ldots \mu_{r}} \omega_{\mu_{1}} \wedge \omega_{\mu_{2}} \wedge \ldots \wedge \omega_{\mu_{r}}
$$

where $\omega_{\mu_{1}}, \omega_{\mu_{2}}, \ldots, \omega_{\mu_{r}}$ are one form bases in $R^{n}$ and $\Sigma^{\mu_{1} \ldots \mu_{r}}$ is given in (12). This shows that $\Sigma^{\mu_{1} \ldots \mu_{r}}$ can be identified with an alternating decomposable $r$-form. It is known that the projective variety of decomposable forms 
is isomorphic to the Grassmann variety of $r$-dimensional linear subspaces in $R^{n}$. In turn, the Grassmann variety is the classifying space for vector bundle structures. These simple observations may motivate one to look for a link between matroid theory and vector bundle formalism.

Fortunately, the mathematicians have already developed the matroid bundle concept [1]-[3]. The central idea in matroid bundles, introduced by MacPherson [1], is to replace tangent spaces in a differential manifold by oriented matroids. Specifically, one starts with a simplicial complex $X$ associated to a differential manifold $B$ by the smooth triangulation $\eta:\|X\| \rightarrow B$. One considers the linear map $f_{\xi}$ :॥ star $\Delta \| U \subset T_{\eta(\xi)}$ such that $f_{\xi}(\xi)=0$, where $\|\Delta\|$ is the minimal simplex of $\|X\|$ containing $\xi \in X$. Then, $f_{\xi}\left\|(\operatorname{star} \Delta)^{0}\right\|$, where $(\operatorname{star} \Delta)^{0}$ are the 0 -simplices of $\operatorname{star} \Delta$, is a configuration of vectors in $T_{\eta(\xi)}$ defining an oriented matroid $\mathcal{M}(\xi)$. (For a more precise definition of matroid bundle, see [1] and [3].)

Suppose we identify the differential manifold $B$ with the world-volume of a $p$-brane. According to our previous discussion one can associate an oriented matroid $\mathcal{M}(\xi)$ at each point $\xi$ of $X$ via the configuration of vectors given by the map $f_{\xi} \|(\operatorname{star} \Delta)^{0}$ ॥. If we consider the oriented matroid $\mathcal{M}(\xi)$ in terms of $(S, \chi)$ with $\chi\left(\mu_{1}, \ldots, \mu_{r}\right) \equiv \operatorname{sign} \Sigma^{\mu_{1} \ldots \mu_{r}}$ we discover that the function $f_{\xi}$ should induce a map

$$
\Sigma^{\mu_{1} \ldots \mu_{r}} \rightarrow \sigma^{\mu_{1} \ldots \mu_{p+1}}(\xi)
$$

where we consider that the rank $r$ of $\mathcal{M}(\xi)$ is $r=p+1$. Note that the formula (15) means that the function $f_{\xi}$ also induces the map $b_{a}^{\mu} \rightarrow v_{a}^{\mu}(\xi)$.

Our last task is to find a mechanism to go from (4) to (5). Consider the expression

$$
F_{a b}^{\mu}=\partial_{a} v_{b}^{\mu}(\xi)-\partial_{b} v_{a}^{\nu}(\xi) .
$$

If the object $F_{a b}^{\mu}$ vanishes, then a solution of (16) is $v_{a}^{\mu}(\xi)=\frac{\partial x^{\mu}}{\partial \xi^{a}}$, where $x^{\mu}$ is in this context a gauge function. In this case, one says that $v_{a}^{\mu}(\xi)$ is a pure gauge. Of course, $F_{a b}^{\mu}$ and $v_{b}^{\mu}(\xi)$ can be interpreted as field strength and abelian gauge potential, respectively. Using the Palatini formalism, the formula

$$
F_{a b}^{\mu}=0
$$

can be imposed in the action (7) as a constraint. In two dimensions, such a formula may be derived from the abelian Chern-Simons action 


$$
S_{C S}=\frac{k}{2 \pi} \int d^{3} \xi \varepsilon^{i j k} v_{i}^{\mu} F_{j k \mu}
$$

The expressions (15) and (17) can be considered as the key bridge to link $p$-branes and matroid bundles. It is worth mentioning that according to the action (7), our results also apply to null $p$-branes.

It is interesting to observe that $\sigma^{\mu_{1} \ldots \mu_{p+1}}(\xi)$ is a decomposable $p+1-$ form. Thus, $\sigma^{\mu_{1} \ldots \mu_{p+1}}(\xi)$ is connected to the Grassmann variety concept associated to a matroid bundle. In the literature this kind of Grassmann variety is called MacPherson's variety. It turns out that the MacPherson variety plays the same role for matroid bundles as the ordinary Grassmann variety plays for vector bundles (see Ref. [3] for details).

One of the attractive features that arises from the above link between matroids and $p$-branes is that the concept of duality becomes part of the $p$-brane structure. The reason for this is that every oriented matroid $\mathcal{M}(\xi)$ has an associated unique dual oriented matroid $\mathcal{M}^{*}(\xi)$ (see Ref. [4], section 3.4) and therefore the identification of $\sigma^{\mu_{1} \ldots \mu_{p+1}}(\xi)$ with the chirotope $\chi$ of $\mathcal{M}(\xi)$ should imply an identification of the dual of $\sigma^{\mu_{1} \ldots \mu_{p+1}}(\xi)$ with the dual chirotope $\chi^{*}$. In order to be more specific in these observations we need to resort on the duality concept in matroid bundle. Unfortunately, it seems that the mathematicians have not yet considered such a concept. Nevertheless, it is tempting to try to outline the main idea. Consider the dual of $\Sigma^{\mu_{1} \ldots \mu_{p+1}}$

$$
{ }^{*} \Sigma^{\mu_{p+2} \ldots \mu_{d+1}}=\frac{1}{(p+1) !} \varepsilon_{\mu_{1} \ldots \mu_{p+1}}^{\mu_{p+2} \ldots \mu_{d+1}} \Sigma^{\mu_{1} \ldots \mu_{p+1}} .
$$

In order to identify ${ }^{*} \Sigma^{\mu_{p+2} \ldots \mu_{d+1}}$ with the corresponding chirotope $\chi^{*}$ we should have

$$
{ }^{*} \Sigma^{\mu_{p+2} \ldots \mu_{d+1}}=\varepsilon^{\hat{a}_{p+2} \ldots \hat{a}_{d+1}} b_{\hat{a}_{p+2}}^{\mu_{p+2}} \ldots b_{\hat{a}_{d+1}}^{\mu_{d+1}},
$$

where the indices $\hat{a}_{p+2, \ldots,} \hat{a}_{d+1}$ run from $p+2$ to $d+1$. Therefore, using (12) and (20) we discover that (19) becomes

$$
\varepsilon^{\hat{a}_{p+2} \ldots \hat{a}_{d+1}} b_{\hat{a}_{p+2}}^{\mu_{p+2}} \ldots b_{\hat{a}_{d+1}}^{\mu_{d+1}}=\frac{1}{(p+1) !} \varepsilon_{\mu_{1} \ldots \mu_{p+1}}^{\mu_{p+2} \ldots \mu_{d+1}} \varepsilon^{a_{1} \ldots a_{p+1}} b_{a_{1}}^{\mu_{1}} \ldots b_{a_{p+1}}^{\mu_{p+1}} .
$$

An important duality property is that the vectors $b_{\hat{a}}^{\mu}$ and $b_{a}^{\mu}$ should satisfy the orthogonality condition (see Ref. [19]) 


$$
b_{\hat{a}}^{\mu} b_{a \mu}=0
$$

In order to make sense of the formula (21) at the level of matroid bundle we need to consider the maps $b_{a}^{\mu} \rightarrow v_{a}^{\mu}(\xi)$ and $b_{\hat{a}}^{\mu} \rightarrow v_{\hat{a}}^{\mu}(\xi)$. In this case (21) becomes

$$
\varepsilon^{\hat{a}_{p+2} \ldots \hat{a}_{d+1}} v_{\hat{a}_{p+2}}^{\mu_{p+2}}(\xi) \ldots v_{\hat{a}_{d+1}}^{\mu_{d+1}}(\xi)=\frac{1}{(p+1) !} \varepsilon_{\mu_{1} \ldots \mu_{p+1}}^{\mu_{p+2} \ldots \mu_{d+1}} \varepsilon^{a_{1} \ldots a_{p+1}} v_{a_{1}}^{\mu_{1}}(\xi) \ldots v_{a_{p+1}}^{\mu_{p+1}}(\xi) .
$$

The next step is to connect (23) with a $p$-brane and its dual. This can be achieved by writing

$$
v_{a}^{\mu}(\xi)=\partial_{a} x^{\mu}(\xi)
$$

and

$$
v_{\hat{a}}^{\mu}(\xi)=\partial_{\hat{a}} x^{\mu}(\xi),
$$

where $x^{\mu}(\xi)$ are $d+1$-scalar fields. But this means that we should have $\xi=\left(\xi^{a}, \xi^{\hat{a}}\right)$ instead of just $\xi=\left(\xi^{a}\right)$. In the context of fiber bundles the coordinates $\xi^{a}$ parametrize locally the base space $B$. Therefore we are forced to identify the coordinates $\xi^{\hat{a}}$ with the fiber $F$ of some bundle $E$ with base space $B$. Fortunately, this kind of scenario is possible if we associate $v_{a}^{\mu}(\xi)$ with the horizontal part $H_{\xi}(E)$ and $v_{\hat{a}}^{\mu}(\xi)$ with the vertical part $V_{\xi}(E)$ of a tangent bundle $T_{\xi}(E)$, where $\xi$ is any point in the total space $E$. In fact in this case we have that if $v_{a}^{\mu}(\xi) \in H_{\xi}(E)$ and $v_{\hat{a}}^{\mu}(\xi) \in V_{\xi}(E)$ then

$$
v_{\hat{a}}^{\mu} v_{a \mu}=0
$$

as required by $(22)$.

Another interesting aspect of the matroid-brane connection is that in matroid theory the concept of duality may be implemented at the quantum level for different $p$-branes. In fact, an important theorem in oriented matroid theory assures that

$$
\left(\mathcal{M}_{1} \oplus \mathcal{M}_{2}\right)^{*}=\mathcal{M}_{1}^{*} \oplus \mathcal{M}_{2}^{*},
$$

where $\mathcal{M}_{1} \oplus \mathcal{M}_{2}$ is the direct sum of two oriented matroids $\mathcal{M}_{1}$ and $\mathcal{M}_{2}$. If we associate $p_{1}$-brane and $p_{2}$-brane to the matroids $\mathcal{M}_{1}$ and $\mathcal{M}_{2}$ respectively, then the corresponding partition functions 


$$
Z_{1}=\int D X \exp \left(S_{p_{1}}^{(3)}\right)
$$

and

$$
Z_{2}=\int D X \exp \left(S_{p_{2}}^{(3)}\right)
$$

should lead to the symmetry $Z=Z^{*}$ of the total partition function $Z=$ $Z_{1} Z_{2}$. Here, the actions $S_{p_{1}}^{(3)}$ and $S_{p_{2}}^{(3)}$ are determined by (7).

Before we make some final comments, let us discuss an extension of the Hodge duality definition (19) suggested my matroid theory. We first observe that the completely antisymmetric object $\varepsilon_{\mu_{1} \ldots \mu_{p+1} \mu_{p+2} \ldots \mu_{d+1}}$, using in (19), is in fact a chirotope associated to the underlaying uniform matroid $U_{n, n}$. It turns out that the matroid $U_{n, n}$ corresponds to the ground set $S=$ $\{1,2, \ldots, n\}$ and bases subset $\mathcal{B}=\{\{1,2, \ldots, n\}\}$, with $n=d+1$. Therefore, there is just one base of $\operatorname{rank} r=n$ in $\mathcal{B}$, namely the set $\{1,2, \ldots, n\}$ itself. Thus, the chirotope $\chi$ associated to this base set reads as $\chi\left(\mu_{1}, \ldots, \mu_{d+1}\right)$ and using (8)-(9) one may verify that $\chi\left(\mu_{1}, \ldots, \mu_{d+1}\right)$ is in fact equal to the density $\varepsilon_{\mu_{1} \ldots \mu_{d+1}}$. The question arises: from many possible chirotopes, why is the chirotope $\varepsilon_{\mu_{1} \ldots \mu_{d+1}}$ used to define duality? An straightforward answer to this question it may say that because the chirotope $\varepsilon_{\mu_{1} \ldots \mu_{d+1}}$ has the required properties for duality. But from the point of view chirotope theory the object $\varepsilon_{\mu_{1} \ldots \mu_{d+1}}$ is just a very particular example of a chirotope. Thus, we arrive to the related question: why do not we use other chirotopes to extend the Hodge duality concept? Let us extend (19) in the form

$$
\ddagger_{\Sigma^{2}}^{\mu_{p+2} \ldots \mu_{r}}=\frac{1}{(p+1) !} \chi_{\mu_{1} \ldots \mu_{p+1}}^{\mu_{p+2} \ldots \mu_{r}} \Sigma^{\mu_{1} \ldots \mu_{p+1}},
$$

where $\chi_{\mu_{1} \ldots \mu_{p+1} \mu_{p+2} \ldots \mu_{r}} \equiv \chi\left(\mu_{1}, . ., \mu_{p+1}, \mu_{p+2}, \ldots, \mu_{r}\right)$ is a chirotope associated to some oriented matroid of rank $r \geq p+1$. In order to emphasize that ${ }^{\ddagger} \boldsymbol{\Sigma}$ is a more general object than ${ }^{*} \boldsymbol{\Sigma}$ let us call ${ }^{\ddagger} \boldsymbol{\Sigma}$ the dualoid of $\boldsymbol{\Sigma}$. Of course, (19) is a particular case of (30), since when $r=d+1$ (30) becomes (19).

As an example of such a dualoid let us consider a 2-brane in $d+1=11$ dimensions and some oriented matroid $\mathcal{M}$ of rank $r=6$. From (30) we have

$$
{ }^{\ddagger} \Sigma^{\alpha \beta \tau}=\frac{1}{3 !} \chi_{\mu \nu \rho}^{\alpha \beta \tau} \Sigma^{\mu \nu \rho} .
$$

This leads to the interesting result that the dualoid $\ddagger \Sigma^{\alpha \beta \tau}$ may also describe a 2-brane in eleven dimensions. In contrast, observe that if instead of (31) we used the traditional Hodge transformation (19) we get that the dual of a 2-brane is a 7 -brane. 
Summarizing, in this brief work we have considered the possibility to connect oriented matroids with $p$-branes. We have shown that makes sense to associate the $p+1$-form $\sigma^{\mu_{1} \ldots \mu_{p+1}}(\xi)$ in the Schild type action (7) with the chirotope $\chi\left(\mu_{1}, \ldots, \mu_{p+1}\right)$ of an oriented matroid. It should emphasized that our procedure is not just a technical translation from $p$-branes to matroid theory which is already interesting, but it is a bridge that may allow to bring many important theorems and concepts in matroid theory to $p$-brane physics. In particular, as a proof of the importance of having established such a bridge, we have shown that the duality concept in matroid theory can be understood as a duality symmetry in the context of $p$-branes. The fact that this symmetry is part of the $p+1$-form/chirotope connection of the Schild type action is a guarantee of having classically such a symmetry in a dynamic context. However, in a quantum context one should be always careful with classical symmetries because of some possible anomalies, but in principle this scenario shows a possible route to investigate the duality matroid symmetry for a $p$-brane at the quantum level.

The question arises whether the present connection between matroids and p-branes may be useful in M-theory itself. Of course, since our analysis applies to any $p$-brane it must also be truth for strings which are part of M-theory structure. But beyond this observation there is a key reason to believe that such a connection may have more implications on M-theory. The key idea is to consider duality as a fundamental principle in M-theory. Just as the equivalence principle in gravity suggested to look for a mathematical structure beyond Euclidean geometry, duality in M-theory seems to require a mathematical structure beyond the mathematical structure usually considered in string theory. Surprisingly, such a mathematical structure seems to be precisely matroid theory as it has come to be evident in references [6]-[9]. The main reason is that duality plays a central role in matroid theory and in fact, in strict sense, matroid theory may even be called a duality theory. If one assumes that in effect matroid theory is the underlaying mathematical structure of M-theory then one should expect new duality properties in M-theory beyond the duality symmetries interrelating the five known superstring theories and $p$-branes. For instance, the string/5-brane duality in ten dimensions [20] may be considered as a particular case of the dualoid described above. In fact, the string/5-brane arises from the field strength $F_{\mu \nu \alpha}=\partial_{[\mu} A_{\nu \alpha]}$ associated to the antisymmetric gauge field $A_{\nu \alpha}$ in ten dimensions. The Hodge dual of $F_{\mu \nu \alpha}$ is

$$
F^{\mu_{1} \mu_{2} \mu_{3} \mu_{4} \mu_{5} \mu_{6} \mu_{7}}=\frac{1}{3 !} \varepsilon^{\mu_{1} \mu_{2} \mu_{3} \mu_{4} \mu_{5} \mu_{6} \mu_{7} \mu_{8} \mu_{9} \mu_{10}} F_{\mu_{8} \mu_{9} \mu_{10}}
$$

Here, $F_{\mu_{1} \mu_{2} \mu_{3} \mu_{4} \mu_{5} \mu_{6} \mu_{7}}=\partial_{\left[\mu_{7}\right.} A_{\left.\mu_{1} \mu_{2} \mu_{3} \mu_{4} \mu_{5} \mu_{6}\right]}$ is the field strength associated to 
the completely antisymmetric gauge field $A_{\mu_{1} \mu_{2} \mu_{3} \mu_{4} \mu_{5} \mu_{6}}$ which in turn implies a 5 -brane structure via the coupling

$$
\sigma^{\mu_{1} \mu_{2} \mu_{3} \mu_{4} \mu_{5} \mu_{6}} A_{\mu_{1} \mu_{2} \mu_{3} \mu_{4} \mu_{5} \mu_{6}} .
$$

Under our considerations the object $\sigma^{\mu_{1} \mu_{2} \mu_{3} \mu_{4} \mu_{5} \mu_{6}}$ can be identified with a chirotope $\chi\left(\mu_{1} \mu_{2} \mu_{3} \mu_{4} \mu_{5} \mu_{6}\right)$ and therefore new duality may arise if instead of (32) we consider the dualoid transformation

$$
{ }^{\ddagger} F^{\mu_{1} \mu_{2} \mu_{3} \mu_{4} \mu_{5} \mu_{6} \mu_{7}}=\frac{1}{3 !} \chi^{\mu_{1} \mu_{2} \mu_{3} \mu_{4} \mu_{5} \mu_{6} \mu_{7} \mu_{8} \mu_{9} \mu_{10}} F_{\mu_{8} \mu_{9} \mu_{10}},
$$

where $\chi^{\mu_{1} \mu_{2} \mu_{3} \mu_{4} \mu_{5} \mu_{6} \mu_{7} \mu_{8} \mu_{9} \mu_{10}}$ may be associated to some oriented matroid of rank ten. Here, ${ }^{\ddagger} F_{\mu_{1} \mu_{2} \mu_{3} \mu_{4} \mu_{5} \mu_{6} \mu_{7}}=\partial_{\left[\mu_{7}\right.} A_{\left.\mu_{1} \mu_{2} \mu_{3} \mu_{4} \mu_{5} \mu_{6}\right]}^{\ddagger}$ is the dualoid field strength, where $A_{\mu_{1} \mu_{2} \mu_{3} \mu_{4} \mu_{5} \mu_{6}}^{\ddagger}$ is the corresponding completely antisymmetric gauge field.

Another, possibility of relating the matroid-brane link with M-theory is via Matrix theory. Some years ago Yoneya [15] showed that it is possible to construct a Matrix theory of M-theory from the Schild type action for strings. The starting point in the Yoneya's work is to consider the Poisson bracket structure

$$
\left\{x^{\mu}, x^{\nu}\right\}=\frac{1}{\gamma} \sigma^{\mu \nu},
$$

where $\gamma$ is an auxiliary field. This identification suggests to replace the Poisson structure by a coordinates operators

$$
\left\{x^{\mu}, x^{\nu}\right\} \rightarrow \frac{1}{i}\left[\hat{x}^{\mu}, \hat{x}^{\nu}\right] .
$$

The central idea is then quantize the constraint

$$
-\frac{1}{\gamma^{2}} \sigma^{\mu \nu} \sigma_{\mu \nu}=T_{p}^{2}
$$

which can be derived from (7) setting $p=1$. According to (35) and (36) one gets

$$
\left(\left[\hat{x}^{\mu}, \hat{x}^{\nu}\right]\right)^{2}=T_{p}^{2} I
$$

where $I$ is the identity operator. It turns out that the constraint (38) plays an essential role in Matrix theory. Extending the Yoneya's idea for strings, 
Oda [14] (see also Ref. [13]) has shown that it is also possible to construct a Matrix model of M-theory from a Schild-type action for membranes. It is clear from our previous analysis of identifying the quantity $\sigma^{\mu \nu}$ with a chirotope $\chi^{\mu \nu}$ that these developments of Matrix theory can be linked with the oriented matroid theory.

Finally, it is known that there are matroids, such as the non-Pappus matroid, which are not realizable. On the other hand, our discussion on the present work has been focused in realizable matroid bundles. This suggests that there must be an extension of $p$-branes of pure combinatorial character. Moreover, it has been proved that matroid bundles have well-defined StiefelWhitney classes [2] and other characteristic classes [21]. In turn, StiefelWhitney classes are closely related to spinning structures. Perhaps, these exciting developments in combinatorial characteristic classes may eventually lead to a matroid/supersymmetry connection.

Acknowledgments: I would like to thank M. C. Marín, J. Saucedo and G. Arreaga for helpful comments.

\section{References}

[1] R. D. MacPherson, "Combinatorial differential manifolds: a symposium in honor of John Milnor's sixtieth birthday," pp. 203-221 in Topological methods on modern mathematics (Stony Brook, NY, 1991), edited by L. H. Goldberg and A. Phillips, Houston, 1993.

[2] L. Anderson and J. F. Davis, "Mod 2 Cohomolgy of Combinatorial Grassmannians," math.GT/9911158.

[3] L. Anderson, New Perspectives. in Geom. Comb. 38, 1 (1999).

[4] A. Bjorner, M. Las Verganas, N. White and G. M. Ziegler, Oriented Martroids, (Cambridge University Press, Cambridge, 1993).

[5] I. M. Gelfand and R. D. MacPherson, Bull. Amer. Math. Soc. 26, 304 (1992).

[6] J. A. Nieto and M.C. Marín, Int. J. Mod. Phys. A18, 5261 (2003), hep-th/0302193. 
[7] J. A. Nieto, Rev. Mex. Fis. 44, 358 (1998).

[8] J. A. Nieto and M. C. Marín, J. Math. Phys. 41, 7997 (2000).

[9] J. A. Nieto, J. Math. Phys. 45, 285 (2004), hep-th/0212100.

[10] A. Schild, Phys. Rev. D 16, 1722 (1977).

[11] J. Gamboa, C. Ramírez and M. Ruiz-Altaba, Nucl. Phys. B 338,143 (1990).

[12] N. Kitsunezaki and S. Uehara, JHEP 0110, 033 (2001), hepth/0108181.

[13] R. Kuriki, S. Ogushi and A. Sugamoto, Mod. Phys. Lett. A14, 1123 (1999), hep-th/9811029.

[14] I. Oda, "Matrix theory from Schild action", hep-th/9801085.

[15] T. Yoneya, Prog. Theor. Phys. 97, 949 (1997), hep-th/9703078.

[16] P. K. Townsend, "Four lectures on M-theory," Proceedings of the ICTP on the Summer School on High Energy Physics and Cosmology, June 1996, hep-th/9612121.

[17] M. J. Duff, Int. J. Mod. Phys. A 11, 5623 (1996), hep-th/9608117.

[18] P. Horava and E. Witten, Nucl. Phys. B 460, 506 (1996).

[19] J. G. Oxley, Martroid Theory, (Oxford University Press, New York, 1992).

[20] M. J. Duff, and J. X. Lu, Class. Quant. Grav. 9, 1 (1992).

[21] D. Biss, "Some applications of oriented matroids to topology," PhD. thesis, MIT, 2002. 